\title{
Elaborate pupils in skates may help camouflage the eye
}

\author{
Sean Youn ${ }^{1,2, *}$, Corey Okinaka ${ }^{1,3, *}$ and Lydia M. Mäthger ${ }^{1, \pm}$
}

\begin{abstract}
The skate Leucoraja erinacea is a bottom-dweller that buries into the substrate with its eyes protruding, revealing elaborately shaped pupils. It has been suggested that such pupil shapes may camouflage the eye, yet this has never been tested. Here, we asked whether skate pupils dilate or constrict depending on background spatial frequency. In experiment 1, the skates' pupillary response to three artificial checkerboards of different spatial frequencies was recorded. Results showed that pupils did not change in response to spatial frequency. In experiment 2, in which skates buried into three natural substrates of different spatial frequencies, such that their eyes protruded, pupils showed a subtle but statistically significant response to changes in substrate spatial frequency. Although light intensity is the primary factor determining pupil dilation, our results show that pupils also change depending on the spatial frequency of natural substrates, which suggests that pupils may aid in camouflaging the eye.
\end{abstract}

KEY WORDS: Crypsis, Spatial frequency, Background, Vision

\section{INTRODUCTION}

The study of camouflage has received an enormous amount of scientific attention, with research encompassing a wide array of study organisms, from animals that actively select habitats to accommodate their fixed body colors and patterns, to those who have changeable camouflage and can adjust their colors and patterns to effectively blend in with their surroundings (e.g. Cott, 1940; Merilaita, 1998; Cuthill et al., 2005; Stevens et al., 2006; Mäthger et al., 2009; Stuart-Fox and Moussalli, 2009; Zylinski and Johnsen, 2011; Hanlon and Messenger, 2018). Interestingly, the majority of this research has concentrated on the animal's body camouflage; what has received scant attention is the question of whether and how animal eyes may be camouflaged, and what role the pupils may play.

Pupils restrict the optical aperture in the eyes of vertebrates and invertebrates. Most pupils respond to light by constricting. This carefully regulates the amount of light entering the eye, and it is a crucial component in optimizing resolution and sensitivity in the retina (Wilcox and Barlow, 1975; Hammond and Mouat, 1985; Land and Nilsson, 2012; Douglas, 2018). A round, dilated pupil is often a very conspicuous structure that stands out, possibly giving away the location of a lurking predator, or camouflaging a prey animal. Circular pupil shapes can be very conspicuous (e.g. Thresher, 1977) because they present clear edges, and it is well known that contrasting edges may be visually easy to detect

\footnotetext{
${ }^{1}$ Marine Biological Laboratory, Bell Center, Woods Hole, MA 02543, USA. ${ }^{2}$ Wiess School of Natural Sciences, Rice University, Houston, TX 77005, USA. ${ }^{3}$ Biological Sciences Division, University of Chicago, Chicago, IL 60637, USA.

${ }^{*}$ These authors contributed equally to this work.

‡Author for correspondence (Imathger@mbl.edu)
}

(D) C.O., 0000-0001-8933-1276; L.M.M., 0000-0002-0603-0345

Received 7 November 2018; Accepted 16 December 2018
(Troscianko et al., 2009). Some animals have unusually shaped pupils, such as the mobile $\mathrm{W}$-shaped pupil of cuttlefish, or the Ushaped pupils that are seen in some skates, rays, fish and squid. Several publications state that these pupils are found in species that spend significant amounts of time camouflaging on the substrate, and that these pupils might help camouflage the eye (Muntz, 1977; Douglas, 2018; Douglas et al., 1998, 2002, 2005). By giving the pupil outline a less regular shape, the likelihood of visual edge detectors recognizing the shape as belonging to an eye would be reduced. However, whether this allows the eye to better blend into its surroundings has never been put to the test.

There are only a handful of publications that report specific eye adaptations and pigment markings that reduce the conspicuousness of the eye (Barlow, 1972; Nilsson and Nilsson, 1983; Neudecker, 1989). Yet, for some animals, eye contact is actively sought, as it is an important indicator of emotional state and intention (Burger et al., 1992). Laboratory and field studies have shown that gaze direction (i.e. whether eye contact is made) is an important feature perceived by animals and that it determines the observer's behavior (Gagliardi et al., 1976; Hennig, 1977; Burger and Gochfeld, 1981; Burghardt and Greene, 1988). In a predator-prey context, eye contact is often actively sought because it is a good way to tell whether one has been detected; if eye contact has been made, detection is more likely.

Therefore, when trying to locate a camouflaged predator or prey, identifying the eyes may be of utmost importance; and, from the perspective of the animal that hides from potential predators or prey, hiding the eyes should be paramount. This should be even more important for animals that generally bury in the substrate with only their eyes protruding, as is true of the little skate Leucoraja erinacea.

Leucoraja erinacea has a very unusual pupil shape that, when seen under bright light, consists of a series of frills (Fig. 1). Under low light, the pupil opens to form an almost circular shape. To date, there are no descriptions in the literature regarding the function of this pupil. Leucoraja erinacea is a nearshore/shallow-water species that inhabits depths to approximately $90 \mathrm{~m}$ at maximum (Robins and Ray, 1986). Their eyes lack cone photoreceptors and therefore color vision, and their rods have a maximum spectral absorbance at around $500 \mathrm{~nm}$ (Dowling and Ripps, 1970; Cornwall et al., 1989). Batoid elasmobranchs implement a range of feeding strategies, including continuous feeding or foraging, ambush predation and filter feeding. Skates are important predators in benthic and demersal communities, preying mostly on fish and invertebrates, and, depending on life history stage, they adopt mixed feeding strategies, including ambushing teleost fish and squid, and searching for smaller invertebrates (McEachran and Musick, 1975; Ajayi, 1982; Ebert et al., 1991; Orlov, 1998; Mabragaña and Giberto, 2007; Jacobsen and Bennett, 2013).

Importantly, although light intensity is the most obvious driving force of the pupillary reflex, it is known that light is not the only factor influencing pupil dilation and constriction (Hess and Polt, 1964; Muntz, 1977; Messenger, 1981; Gamlin et al., 1998; Barbur et al., 2002; Einhäuser et al., 2007; Bradley et al., 2008; Douglas, 2018). For this reason, it may not be surprising to find that animals, 

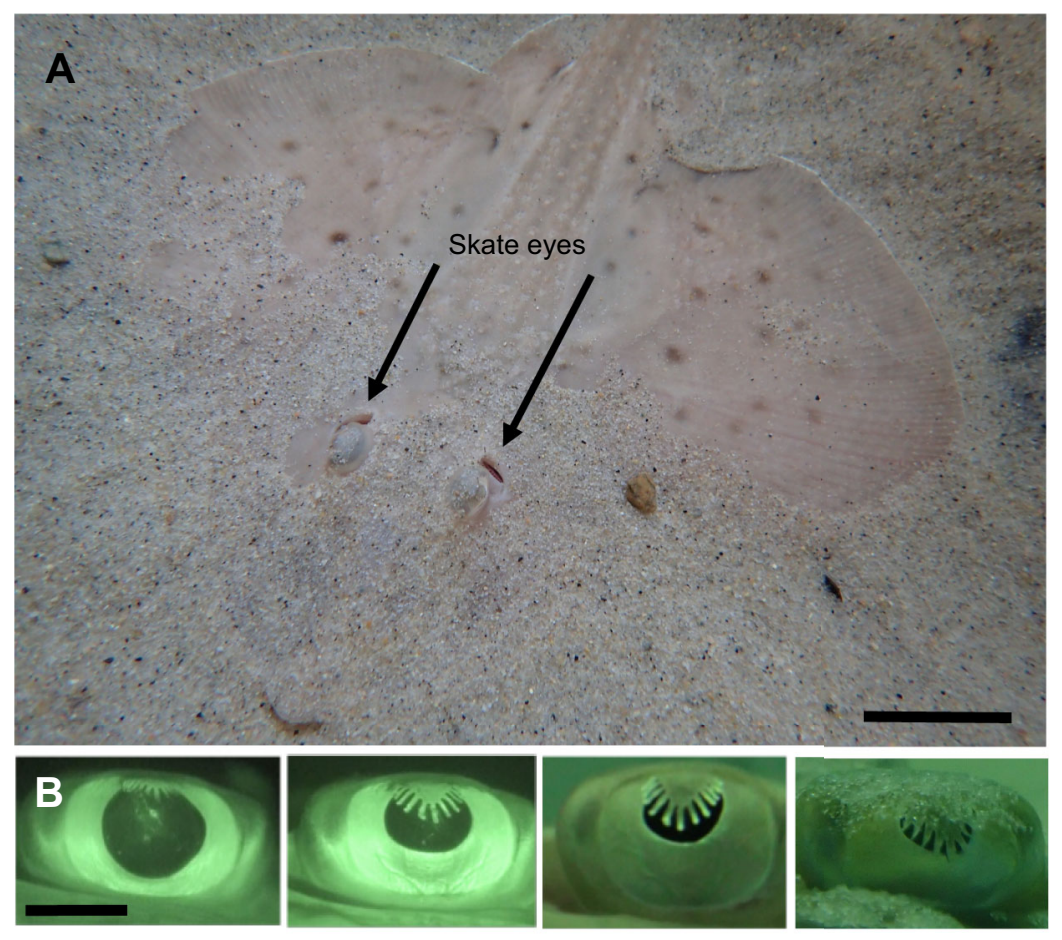

Fig. 1. Eyes of the skate Leucoraja erinacea. (A) A skate partially buried in sand. Scale bar: $8 \mathrm{~cm}$. (B) Close-ups of skate eyes showing the range of dilation/constriction states from fully dilated (left) to near-constricted (right), revealing an elaborate, finger-like shape. Scale bar: $1 \mathrm{~cm}$. especially those with elaborate pupil shapes, actively use their pupils for non-vision-related tasks, such as camouflage.

The goal of our study was to test whether the pupil may function in camouflaging the eye. We conducted two experiments. In the first, we presented skates with checkerboards of different spatial frequencies. This technique has been extensively used to study cuttlefish and fish camouflage. By presenting animals with backgrounds that have known spatial frequencies, such as a blackand-white checkerboard, an animal that has an innate camouflage response will do its best to attempt to respond to this checkerboard accordingly, and this type of behavioral assay has proven to be very robust (e.g. Ramachandran et al., 1996; Mäthger et al., 2006; Kelman et al., 2007; Barbosa et al., 2007, 2008; Allen et al., 2009, 2010; Buresch et al., 2015). In the second experiment, we presented skates with natural substrates that allowed animals to bury with only their eyes protruding. If the pupil in L. erinacea fulfills a camouflage function, we would expect to see the pupil change in response to changes in spatial frequency of the surrounding habitat.

\section{MATERIALS AND METHODS}

\section{Animals}

The little skate Leucoraja erinacea (Mitchill 1825) can be found off the coast of Woods Hole, MA, USA. Animals are regularly collected, as well as raised in captivity. All animals used for these experiments were adults; length measurements, from nose to tip of tail, were between 25 and $43 \mathrm{~cm}$. They were kept at $12-15^{\circ} \mathrm{C}$. For the first experiment, eight animals (two females, six males, measuring between 25 and $33 \mathrm{~cm}$ ) were kept in separate partitions, measuring $75 \times 43 \times 40 \mathrm{~cm} \quad($ length $\times$ width $\times$ height $)$, within a holding tank. Because these experiments took several weeks to complete, and skates are better kept in a larger space, we modified our holding tanks for the second experiment. For the second experiment, 14 skates (six females, eight males, measuring between 31 and $43 \mathrm{~cm}$ ) were kept as a group in a large holding tank $(1.2 \times 3.6 \times 0.40 \mathrm{~m}$, length $\times$ width $\times$ height). Individual skates were recognized by sex (males and females are easy to tell apart), specific body markings and size. Skates were fed five times a week (variation of squid, butterfish and capelin), and all were cared for and experiments were conducted in accordance with the regulations of the Marine Biological Laboratory Institutional Animal Care and Use Committees.

The methodology for experiments 1 and 2 differed slightly. We made specific adjustments because skate size differed between experiments (see above for animal sizes used in each experiment). Also, animals were presented with different types of substrate that required a different amount of space around the animal; e.g. experiment 2 required more space because animals buried into the substrates. In these types of basic behavioral experiments, which investigate behavioral responses to changes in external visual stimuli, such methodological differences are at times necessary but they do not affect the outcome of results. For example, similar methodological differences between experiments have been reported by others, with the same behavioral response to changes in external visual stimuli, such as spatial frequency (see Barbosa et al., 2007; Allen et al., 2009; note that cuttlefish showed the same responses to spatial frequency, irrespective of tank size or experimental methodology).

\section{Experimental setup}

The experimental setup consisted of a glass tank that was placed inside an improvised dark room constructed from an ice-fishing tent (Shappell DX4000) surrounded by metal walls and black plastic sheeting that ensured there was no stray light entering the experimental setup. All video recording was done using a Sony HDR-XR550VEB video camera, equipped with night-shot, so that skate eyes could be recorded under low light. All experiments were performed between 09:00 and 17:00 h. Experiments 1 and 2 required different materials, and methods were therefore slightly different.

\section{Experiment 1: checkerboard experiment}

The experimental tank measured $90 \times 30 \times 40 \mathrm{~cm}$ (length $\times$ width $\times$ height $)$, and was lined on the outside, i.e. all four tank sides and a lid, which was placed on top of the tank during experiments, with a white diffuser filter (Lee Filters Half White Diffusion) and a blue-green 
filter (Roscolux \#374), to provide skates a diffuse light field similar to a natural underwater habitat, and, additionally, to shield animals from unwanted visual stimuli, e.g. the experimenter moving. The light source, placed approximately $15 \mathrm{~cm}$ above the tank, consisted of two side-by-side custom-made light boxes that illuminated the entire tank evenly. Each box was equipped with a Philips $60 \mathrm{~W}$ compact fluorescent bulbs and a Lee Full White Diffusion filter. The light intensities were measured with an Extech EasyView 30 light meter with an irradiance probe, which was placed on the bottom of the tank to collect downwelling light. The light intensity across the tank varied by less than 5\%; at full illumination, the intensity was approximately $700 \mathrm{~lx}$. Note that using a lux meter, instead of a full spectrum radiometer, is convenient and sufficient for these experiments, because skates are monochromats with a maximum sensitivity at around $500 \mathrm{~nm}$, which overlaps with the maximum sensitivity of the Extech light meter. Three light intensities were created using neutral density filters (combination of $0.15 \mathrm{ND}$ and $0.6 \mathrm{ND}$, Lee Filters). In a separate experiment, we verified the light intensities required for pupils to be dilated, semi-constricted and near-constricted; these were 0.68, 10.9 and $43.751 x$, respectively. The light-emitting pupil area of a fully constricted pupil is difficult to analyze; therefore, we chose a pupil constriction state that was near-constricted. The pupil dilation and constriction states, and required light intensities, were obtained during an unrelated experiment.

We created three black-and-white (i.e. $0,0,0$ and 255,255,255 RGB) checkerboard substrates of different spatial frequencies (Fig. 2A). To create the three spatial frequencies, we measured the anterior-posterior dimension of all skates' eyes. These eye dimension values, indicated as E.D. in Fig. 2A, were used to make the checkerboards. The large checkerboard had a check size of $100 \%$ of this distance, the medium checkerboard had a check size of $50 \%$, and the small checkerboard had a check size of $25 \%$. Not all skates had the same eye size; thus we made three sets of checkerboards to accommodate all skate eye sizes. The skates were organized into three groups based on eye size. Group 1: four skates had the same eye size to within less than $1 \mathrm{~mm}$ (mean \pm s.d. $=6.96 \pm 0.24 \mathrm{~mm}$ ). Their three checkerboards had check sizes of 7, 3.5 and $1.75 \mathrm{~mm}$, respectively. Group 2: three skates had larger eyes (mean \pm s.d. $=7.87 \pm 0.16 \mathrm{~mm}$ ), and their three checkerboards had check sizes of 8,4 and $2 \mathrm{~mm}$, respectively. Group 3: one animal had the largest eyes $(10.06 \mathrm{~mm})$, and its three checkerboards had check sizes of 10, 5 and $2.5 \mathrm{~mm}$, respectively. All checkerboards were printed on regular matte printing paper and laminated to be waterproof (matte laminate). Because all checks were made up of the same black and white shades, the overall reflectance of all substrates was the same.
A

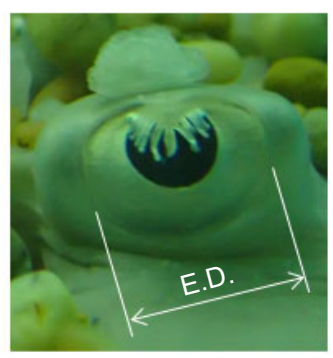

B
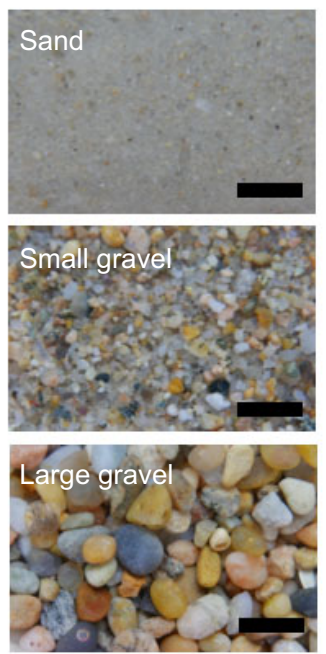
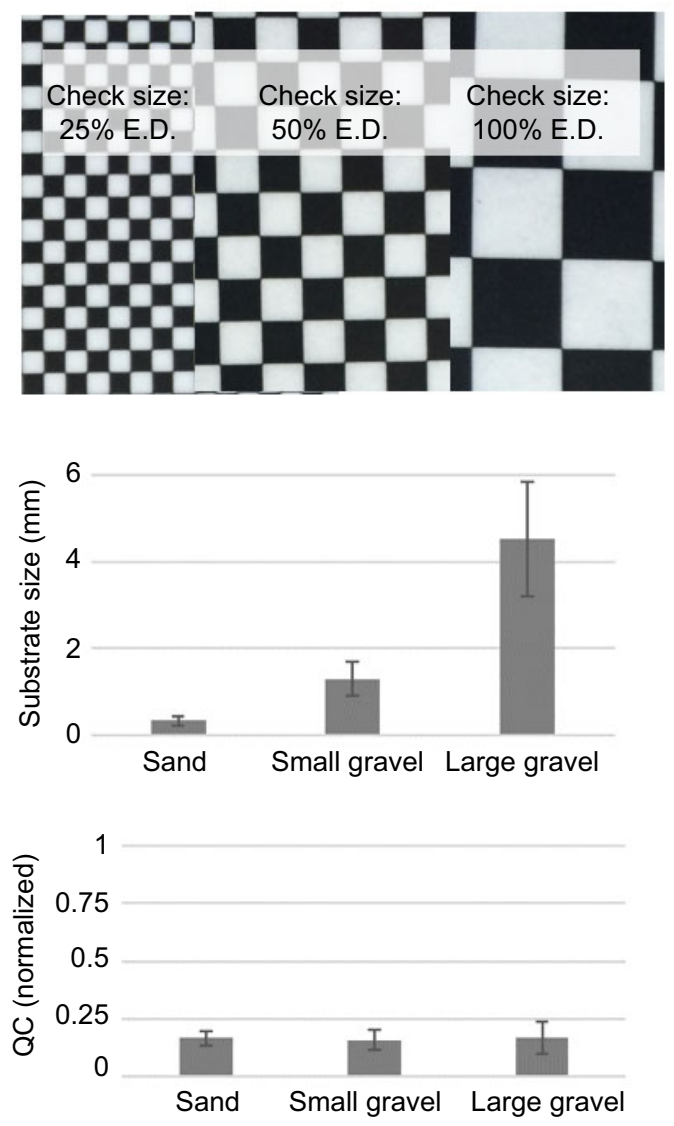

Fig. 2. Substrates used in experiments 1 and 2.

(A) Checkerboard substrates for experiment 1 were created relative to skate eye dimensions. The largest checkerboard used a check size/length equivalent to the anterior-posterior dimension of the skate's eye; the medium checkerboard's size was $50 \%$ of the eye dimension; and the small checkerboard $25 \%$ of the eye dimension (E.D.). Skate eye and checkerboard images are not the same scale. (B) Images of the natural substrates used in experiment 2. Scale bars: $1 \mathrm{~cm}$. Top right: substrate size (diameter of substrate objects, in $\mathrm{mm}$ ) of the three natural substrates used in experiment 2. Bottom right: quantum catch (QC, a measure of the relative intensity at which an animal visually perceives a given object) of the three natural substrates used in experiment 2 (note that there was no significant difference between substrate size QC; see Results for details). (C) Comparison of the size ranges used in experiments 1 and 2 . Black dots, large check/particle size; gray dots, medium check/particle size; white dots, small check/ particle size. Relative eye dimensions for the checkerboards are $100 \%, 50 \%$ and $25 \%$ E.D., respectively; relative eye dimensions for the natural substrates are $45 \%, 0.13 \%$ and $0.03 \%$ E.D., respectively. See Materials and Methods for more detail.

C

\begin{tabular}{|l|l|l|l|l|l|l|ll|l|l|l|}
\hline & 1 & 2 & 3 & 4 & 5 & 6 & 7 & 8 & 9 & $10 \mathrm{~mm}$ \\
\hline & 0 & 0 & 0 & 0 & 0 & & & 5 & & & 1: Check substrate sizes \\
\hline 0 & 0 & & & & & & & & & & 2: Natural substrate sizes \\
\hline
\end{tabular}


Checkerboard substrates were placed on the bottom of the aquarium. We also placed checkerboards on the inside of the tank walls to enhance the three-dimensionality of the otherwise flat substrate. Checkerboard walls were $10 \mathrm{~cm}$ high and placed along the back and side walls, leaving the front wall open for filming. There was no particular order in which the skates were placed on their respective checkerboards. For each experiment, the lowest light intensity was set up first and filters were progressively removed to proceed to the next brighter light intensity. This proved more feasible than sliding filters into the light boxes to reduce light intensity. For each light setting, skates were given 30 min acclimation time, to ensure that the skate was calm and resting on the aquarium floor, ready for the experiment. During this time, they were video recorded for $10 \mathrm{~s}$ every $5 \mathrm{~min}$, so that the pupil dilation process could be documented. After 10-15 min, there was usually no further change in pupil dilation. After a total of $30 \mathrm{~min}$ acclimation, the skates were filmed for $2 \mathrm{~min}$. We then removed the neutral density filters to proceed to the next brighter light setting and allowed another $30 \mathrm{~min}$ adaptation, during which the pupil dilation was recorded as described before. After $30 \mathrm{~min}$, the skate was video recorded for $2 \mathrm{~min}$. This procedure was repeated for each light intensity.

The video camera was positioned horizontally, and perpendicular to the long axis of the skate, i.e. looking directly at the skate's eye from the side, so that we were able to document the entire pupillary area during dilation and constriction. Note that when the eye is seen from angles other than perpendicular to the skate's long axis, the apparent total pupil area becomes smaller, so it was crucial to maintain this camera angle throughout the experiment. The camera angle was set to be perpendicular to the aquarium glass to avoid error caused by diffraction. While filming, the filters on the outside of the tank were lifted enough to accommodate the camera. Because of the tank dimensions, skates usually positioned themselves with their long axis perpendicular to the camera, in which case both eyes, i.e. the eye that was being filmed and the eye on the opposite side, were in a straight line, perpendicular to the camera. If the opposite eye was ahead or behind the eye being filmed, the skate was not aligned correctly. If skates had to be re-aligned, animals generally allowed us to move them and remained settled. Every skate was recorded when settled on its custom-made large, medium and small checkerboards at all three light intensities.

\section{Experiment 2: natural substrates experiment}

The experimental tank for this experiment measured $60 \times 60 \times 45 \mathrm{~cm}$ (length $\times$ width $\times$ height), placed into the same dark-room set-up described above. A larger tank was needed to allow skates to move about and bury themselves. Consequently, the overhead light source consisted of three custom-made light boxes (lined with LE Flexible Strip, SMD 2835 Daylight White LEDs) to provide even illumination across the entire tank (less than 5\% variation; verified by Extech EasyView 30 light meter; at full illumination, the light intensity was measured to be approximately $500 \mathrm{~lx}$ ). The tank was lined with the same filters as described for experiment 1 . Neutral density filters (combination of $0.15 \mathrm{ND}$ and $0.6 \mathrm{ND}$, Lee Filters) were used to obtain three light intensities that were previously verified to result in three distinct pupil dilation states: fully dilated, semi-constricted and near-constricted. These light intensities were $0.012,1.95$ and $31.25 \mathrm{~lx}$, respectively. Skate sizes were similar for this experiment, yielding similar anterior-posterior eye dimensions (mean: 9.99 mm; 0.11 s.d.).

Three natural substrates were collected locally (Fig. 2B): (1) sand, mean \pm s.d. grain size $=0.33 \pm 0.11 \mathrm{~mm}$, which corresponded to $0.03 \%$ of the anterior-posterior eye dimension (indicated as E.D. in
Fig. 2B); (2) small gravel, mean \pm s.d. grain size $=1.30 \pm 0.40 \mathrm{~mm}$, which corresponded to $0.13 \%$ of this eye dimension; and (3) large gravel, mean \pm s.d. grain size $=4.5 \pm 1.32 \mathrm{~mm}$, which corresponded to $45 \%$ of this eye dimension. These substrates are typical of the sandy/ pebbly habitats in which skates are naturally found. ImageJ (National Institutes of Health, Bethesda, MD, USA) was used to determine grain size (for each substrate, 100 particles were measured to determine average substrate size). In Fig. $2 \mathrm{C}$, we show substrate sizes for experiments 1 and 2 side by side. Note that although there was a difference in the size ranges between the two experiments, the particle/check sizes overlap and complement each other, providing a larger overall tested particle size range.

To ensure that skates responded to the spatial frequency of the natural substrates and not to variation in overall reflectance, we measured spectral reflectance of the three natural substrates using a spectrometer (QE65000, Ocean Optics) to test whether overall reflectance was acceptably similar. A $200 \mu \mathrm{m}$ fiber (400-UV-VIS, Ocean Optics) was held by a clip approximately $8 \mathrm{~mm}$ from the substrate at an angle of $30 \mathrm{deg}$; the illumination consisted of the light source that was used for the behavioral trials (see above). A diffuse reflection standard (WS-1-SL, Ocean Optics), was used to standardize measurements. All measurements were taken in water. For each substrate, 70 measurements were taken. After the relative reflectance spectra were obtained, they were transformed into quantum units (divided by wavelength) and the number of photons $(N)$ absorbed by a skate photoreceptor was calculated. This is given by:

$$
N=\int(1-\exp (-k S(\lambda) l)) \times R(\lambda) \mathrm{d} \lambda
$$

after Warrant (2004), where $S(\lambda)$ is the spectral sensitivity of the visual pigment (500 nm; from Dowling and Ripps, 1970; Cornwall et al., $1989), R(\lambda)$ is the spectral composition of the light reflected from the substrate [both $S(\lambda)$ and $R(\lambda)$ integrated over $400-650 \mathrm{~nm}$ ], $l$ is the length of the photoreceptor $(67 \mu \mathrm{m}$; from Ripps and Dowling, 1990) and $k$ is the quantum efficiency of transduction $\left(0.037 \mu \mathrm{m}^{-1}\right.$; Warrant and Nilsson, 1998). The spectral sensitivity of the visual pigment was calculated using a template provided by A. Kelber, Lund, Sweden (based on Stavenga et al., 1993).

The three natural substrates yielded closely similar quantum catches; the following values are given as normalized values (on a scale of $0-1,0$ being the quantum catch for a pure black object, 1 being the quantum catch for a pure white object): large gravel mean \pm s.d. quantum catch: $0.167 \pm 0.072$ (range: 0.046-0.472); small gravel mean \pm s.d. quantum catch: $0.158 \pm 0.046$ s.d. (range: 0.079 0.328 ); and sand mean \pm s.d. quantum catch: $0.164 \pm 0.033$ s.d. (range: 0.072-0.261) (Fig. 2B). A one-way ANOVA comparing the means of all three quantum catches (i.e. each of the substrates, as seen by a skate) revealed that there was no significant difference between the substrates $\left(F_{2,207}=0.54, P>0.5\right)$.

The bottom of the experimental tank was covered with substrate to a depth of approximately $5 \mathrm{~cm}$. Because the skates moved the substrates sufficiently, resulting in a three-dimensional surface, no walls were used for these substrates. Skates were placed into the experimental tank set-up at the lowest light intensity $(0.012$ lx). Compared with experiment 1 , skates needed a longer acclimation time to settle; thus, animals were given 50 min to acclimate. Next, skates were video recorded for 2 min (same methods as used in experiment 1 ). Neutral density filters were then removed to obtain the next brightest light intensity $(1.95 \mathrm{~lx})$, followed by $50 \mathrm{~min}$ acclimation time and 2 min video recording. The same was repeated for the highest light intensity (31.25 lx). Once all skates were filmed 
at all light intensities on one substrate, we switched to the next substrate and repeated this procedure.

\section{Statistical analysis}

From the 2-min video clips taken at each light intensity, we extracted four to five images at intervals of at least $10 \mathrm{~s}$ apart (as these were live animal experiments, we had to be flexible regarding the number of images we were able to obtain and the time between images). Using these images, we measured pupil dilation/constriction by determining pupil area $\left(\right.$ in $\mathrm{mm}^{2}$ ). This was done by tracing the exact outline of the pupil in ImageJ. To test for statistical significance, we used these pupil area measurements in a one-way ANOVA, with an alpha value of 0.05 .

\section{RESULTS \& DISCUSSION}

Experiment 1: checkerboard experiment

For each light intensity, skates maintained almost the same pupil area, irrespective of check size (Fig. 3A). There was no statistical difference in pupil area on different checkerboards at each of the light intensities (one-way ANOVA; $0.681 \mathrm{~lx}: F_{2,117}=0.65, P>0.5$; 10.9 lx: $F_{2,117}=0.34, P>0.7$; 43.75 lx: $\left.F_{2,117}=1.48, P>0.2\right)$. Pupil area only changed in response to light intensity, with a significant difference between $0.68,10.9$ and 43.75 lx. Because pupil area was the same on all check sizes irrespective of light intensity, all data for

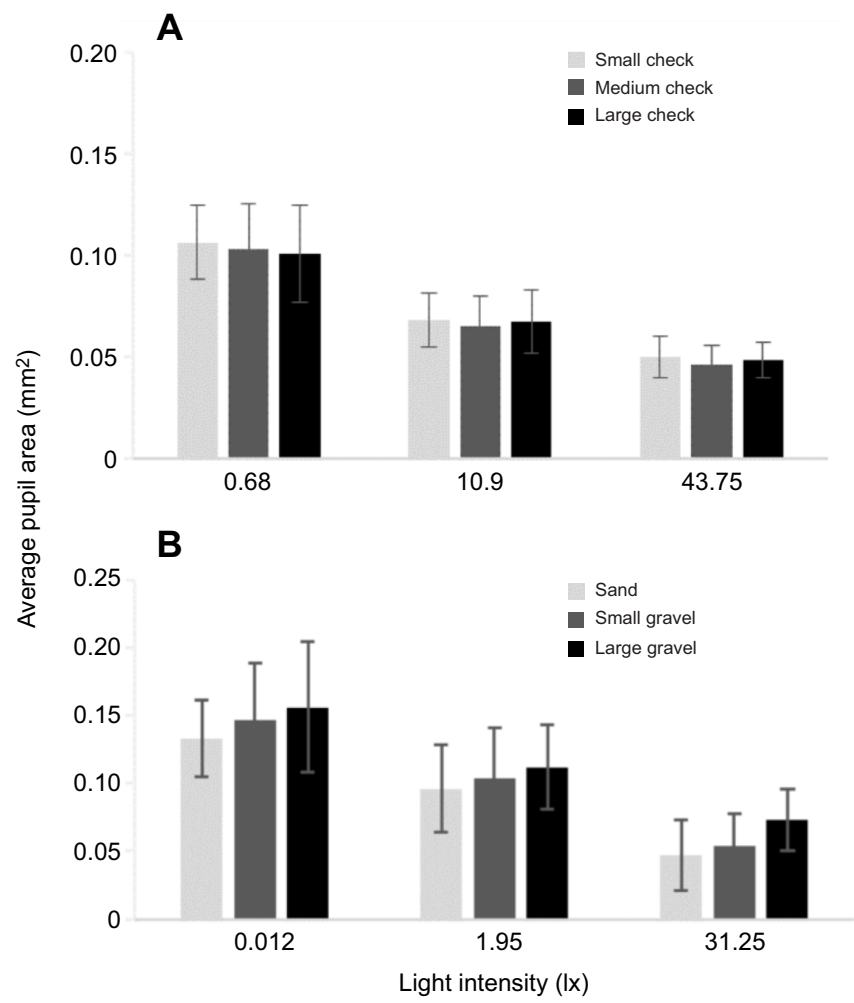

Fig. 3. Average pupil areas on experimental substrates. (A) Experiment 1: pupil dilation for skates placed on artificial checkerboards. The skate pupil constricted with increasing light intensity but did not respond to changes in spatial frequency of the checkerboards (there was no significant difference between check sizes; there was a significant difference between light intensities; see Results for details). (B) Experiment 2: pupil dilation for skates allowed to bury in natural substrates, with only their eyes protruding. In addition to reacting to light intensity, the skate pupil constricted/dilated in response to the spatial frequency of the substrate: the smaller the substrate spatial frequency, the more constricted the pupil. This effect was statistically significant (see Results for details). Note that different dilation:constriction ratios in $A$ and $B$ are due to skate size differences. each light intensity were averaged, and a one-way ANOVA comparing the means on all three light intensities revealed that there was a significant difference in pupil area measured at different light intensities $\left(F_{2,357}=378.5, P<0.0001\right)$.

\section{Experiment 2: natural substrates experiment}

As in experiment 1, pupil area changed in response to light intensity, with a significant difference between $0.012,1.95$ and $31.25 \mathrm{~lx}$ (oneway ANOVA; sand: $F_{2,165}=127.7, \quad P<0.0001$; small gravel: $F_{2,165}=100.4, P<0.0001$; large gravel: $\left.F_{2,165}=80.3, P<0.0001\right)$. In contrast to experiment 1 , skate pupil dilation also depended on substrate spatial frequency (Fig. 3B). Given the same light intensity, the pupils were more constricted on substrates with smaller spatial frequency, and more dilated on substrates with larger spatial frequency. This effect was statistically significant (one-way ANOVA; $0.012 \mathrm{~lx}: F_{2,165}=4.98, P=0.0078 ; 1.95 \mathrm{~lx}: F_{2,165}=3.2$, $P=0.043$; $\left.31.25 \mathrm{~lx}: F_{2,165}=17.56, P<0.0001\right)$.

\section{Conclusions}

While it has been suggested that pupils may aid in camouflaging the eye (Muntz, 1977; Douglas, 2018; Douglas et al., 1998; 2002; 2005), this has never been tested. The two experiments reported here present evidence that the elaborate pupil shape of $L$. erinacea may indeed serve a camouflage function. When skates were placed on artificial backgrounds of different spatial frequencies, their pupils only dilated/constricted in response to light intensity - not check size. When placed on natural substrates that allowed burying, so that only the eyes protruded, the skates' pupils dilated/constricted in response to the spatial frequency of the substrate as well as the light intensity. Although these findings seemingly contradict each other, when considered in the context of how camouflage works, the possible rationality behind this emerges. Object recognition is subject to a multi-step process that begins with the detection of the object by the observer's visual system. This depends on the visual system of the observer and the physical characteristics of both the object and the background against which the object is seen. Background characteristics are paramount in the context of camouflaged objects (Thayer, 1909; Longley, 1916; Longley, 1917; Cott, 1940; Endler, 1981, 1984; Crook, 1997a, b; Barry and Hawryshyn, 1999; Marshall, 2000; Vorobyev et al., 2001; Marshall et al., 2003). Going back to experiment 1 , in which the skates were placed on artificial backgrounds that did not permit burying, the background against which an observer would see the skates' eyes was not the checkerboard substrate but the skate's head and body (because the skate was not buried). Consequently, unless the skate's head and body colors or patterns change in response to the spatial frequencies of the checkerboard substrates, there may be no reason for the skate's pupil to react to the different background spatial frequencies. Skates do not have the fast color-changing abilities we see in some teleost fish and cephalopods (Parker and Porter, 1934; Waring, 1963; Hanlon and Messenger, 2018), and, although elasmobranchs are capable of subtle color tone and pattern changes (Parker and Porter, 1934; Parker, 1936; Visconti et al., 1999), these changes generally occur over the course of many hours. The short duration of our experiments was likely not long enough for any color or pattern changes to occur. Notably, we did not observe any color or pattern changes in our experiments.

In experiment 2 , burying behavior was permitted, and all skates buried themselves. In this situation, the eyes were seen against the natural substrate, rather than the skate's body; thus, the fact that the skate's pupil changed with changes in background spatial frequency is entirely rational. 
It should be reiterated here that the methodology for experiments 1 and 2 differed slightly because animal sizes differed between experiments, and animals were presented with substrates that required a different amount of space around the animal (i.e. natural substrates required a larger tank so animals could move to bury themselves). Such methodological differences are at times needed, but for these types of basic behavioral experiments that test a response to an external visual stimulus, they do not affect the results (see differences in methodology reported by e.g. Barbosa et al., 2007; Allen et al., 2009).

In order to understand the possible rationale behind the skate pupillary movements we report here, it is necessary to briefly discuss some fundamental camouflage principles. A range of colors and patterns have evolved in many animals for the sole purpose of reducing the risk of detection, with the ultimate goal of evading predation (e.g. Endler, 1978; Stevens and Cuthill, 2006; Stevens and Merilaita, 2009; Troscianko et al., 2009; Stevens and Merilaita, 2011). To avoid detection, several cryptic mechanisms are deployed by animals; briefly, they include background matching, self-shadow concealment, obliterative shading, disruptive coloration, flickerfusion camouflage, and distractive markings (Stevens and Merilaita, 2009). While avoiding detection would be the best option, this is often hard to achieve in nature. Animals move between habitats and are therefore frequently faced with changing background characteristics, yet most animals have only one or a limited number of body colors and patterns available to them. Additionally, some structures - e.g. the eyes - are impossible to conceal to a level that would completely eliminate detection; the pupil, by definition, is a dark hole for light to enter so that vision can ensue. As a result, many animals have solved this problem by having physical characteristics that reduce the likelihood of recognition, for example, by creating false contours or distractive markings, or by disguising the body's true outline using disruptive coloration (Cuthill and Székely, 2009). These forms of camouflage are most likely aimed at fooling a specific observer's perceptual processes, for example, their visual system's color or polarization vision ability, or their edge detection mechanisms (Stevens and Merilaita, 2009; Troscianko et al., 2009; Stevens and Merilaita, 2011). It is likely that the camouflage mechanisms underlying the elaborate pupil shape of the skate fall under this category; specifically, the irregular pupil outline creates an edge pattern that, compared with a conventional circular pupil pattern, would be much harder to detect. Because skates like to bury into the substrate to practice their various predatory behaviors (e.g. active foraging, sit-and-wait tactics), the eye is usually the only part of the animal that is visible. The iris structures that form the elaborate pupil shapes are lined with silvery reflectors, similar to the ones we see in cephalopod eyes (Denton and Land, 1971; Mäthger et al., 2009), providing the animal with the ability to match the surrounding natural background in color and brightness. However, as discussed in illustrative detail by Cott (1940), animal eyes are intrinsically difficult to conceal because of their highly regular structures that do not blend well into the usually irregular spatial features of natural backgrounds. Elaborate pupil shapes, such as those of skates, may therefore aid camouflage by introducing irregular shapes and lines that break up the otherwise conspicuous outline of the pupil, so that it may reduce the likelihood of being recognized as part of an eye that belongs to a potential predator (or prey). Additionally, to help achieve the required level of camouflage on a range of natural substrates, it would seem advantageous to adjust pupil dilation and constriction relative to background spatial frequency, which is what we found in the present study. However, in skates, these dilation and constriction changes were small, and did not exactly match the spatial frequencies of the surrounding backgrounds. Moreover, directionality of viewing also plays an important role in that the skate pupil will be seen from a range of different viewing angles, depending on the observer's lifestyle, potentially requiring a finetuned system for this to be fully effective for camouflage. More work is clearly needed to fully evaluate the contribution of pupillary movements in camouflage on backgrounds of different spatial frequencies. For example, the size ranges of the natural and artificial substrates did not completely overlap. The artificial checkerboard substrates concentrated on higher spatial frequencies, whereas the natural substrates concentrated on lower spatial frequencies. It would be valuable to study the pupillary response at the lower spatial frequency range, which is more relevant to the lifestyle of these animals.

Obviously, one crucial purpose of a pupil is to regulate the amount of light entering the eye (e.g. Land and Nilsson, 2012; Douglas, 2018). By adjusting the pupil according to the spatial frequency of the background, it seems that the skate retina is able to handle a small range of light throughput around a given light intensity. Skates have an unusual retina in that it contains only rods (no cones), yet, the rods' physiology changes depending on light intensity, from a rod-typical sluggish response to a much faster response, typical for cones (Ripps and Dowling, 1990; Dowling, 2013). Thus, the skates' visual system may be particularly well adapted to allow the pupil to depart slightly from its optimal dilation/constriction state to a given light intensity.

In conclusion, by compromising retinal illumination, the animal may gain a critical camouflage advantage. Although the pupil does not completely match the spatial frequency of the background, compared with not responding to spatial frequency at all, it may be better camouflaged with just a small spatial frequency adaptation. Future work is needed to investigate whether these small adaptations make visual detection less likely, and whether this truly helps camouflage the eye.

\section{Acknowledgements}

S.Y. and C.O. were undergraduate student interns. The authors would like to thank Dan-Eric Nilsson for stimulating discussions, and the staff of the Marine Resources Center at the Marine Biological Laboratory for assistance with skates.

\section{Competing interests}

The authors declare no competing or financial interests.

\section{Author contributions}

Conceptualization: L.M.M.; Methodology: L.M.M.; Formal analysis: L.M.M.; Investigation: S.Y., C.O., L.M.M.; Resources: L.M.M.; Writing - original draft: L.M.M.; Writing - review \& editing: S.Y., C.O., L.M.M.; Supervision: L.M.; Project administration: L.M.M.; Funding acquisition: L.M.M.

\section{Funding}

This work was funded by awards from the Marine Biological Laboratory (specifically, the Hermann Foundation Award, Joan Ruderman Fund Award, Grass Foundation Fund Award and Neal Cornell Career Development Award), as well as a University of Chicago Metcalf Fellowship to C.O.

\section{References}

Ajayi, T. O. (1982). Food and feeding habits of Raja species (Batoidei) in Carmarthen Bay, Bristol Channel. J. Mar. Biol. Assoc. UK 62, 215-223.

Allen, J. J., Mäthger, L. M., Barbosa, A. and Hanlon, R. T. (2009). Cuttlefish use visual cues to control 3-dimensional skin papillae for camouflage. J. Comp. Physiol. A 195, 547-555.

Allen, J. J., Mäthger, L. M., Buresch, K. C., Fetchko, T., Gardner, M. and Hanlon, R. T. (2010). Night vision by cuttlefish enables changeable camouflage. J. Exp. Biol. 213, 3953-3960.

Barbosa, A., Mäthger, L. M., Chubb, C., Florio, C., Chiao, C.-C. and Hanlon, R. T. (2007). Disruptive coloration in cuttlefish: a visual perception mechanism 
that regulates ontogenetic adjustment of skin patterning. J. Exp. Biol. 210, 1139-1147

Barbosa, A., Mäthger, L. M., Buresch, K. C., Kelly, J., Chubb, C., Chiao, C.-C. and Hanlon, R. T. (2008). Cuttlefish camouflage: the effects of substrate contrast and size in evoking uniform, mottle or disruptive body patterns. Vision Res. 48, 1242-1253.

Barbur, J. L., Prescott, N. B., Douglas, R. H., Jarvis, J. R. and Wathes, C. M. (2002). A comparative study of stimulus-specific pupil responses in the domestic fowl (Gallus gallus domesticus) and the human. Vision Res. 42, 249-255.

Barlow, G. W. (1972). The attitude of fish eye-lines in relation to body shape and to stripes and bars. Copeia 1972, 4-12.

Barry, K. L. and Hawryshyn, C. W. (1999). Spectral sensitivity of the Hawaiian saddle wrasse, Thalassoma duperrey, and implications for visually mediated behaviour on coral reefs. Environ. Biol. Fishes 56, 429-442.

Bradley, M. M., Miccoli, L., Escrig, M. A. and Lang, P. J. (2008). The pupil as a measure of emotional arousal and autonomic activation. Psychophysiology 45 602-607.

Buresch, K. C., Ulmer, K. M., Akkaynak, D., Allen, J. J., Mäthger, L. M., Nakamura, M. and Hanlon, R. T. (2015). Cuttlefish adjust body pattern intensity with respect to substrate intensity to aid camouflage, but do not camouflage in extremely low light. J. Exp. Mar. Biol. Ecol. 462, 121-126.

Burger, J. and Gochfeld, M. (1981). Discrimination of the threat of direct versus tangential approach to the nest by incubating herring and great black-backed gulls. J. Comp. Physiol. Psychol. 95, 676-684.

Burger, J., Gochfeld, M. and Murray, B. G. (1992). Risk discrimination of eye contact and directness of approach in black iguanas (Ctenosaura similis) J. Comp. Psychol. 106, 97-101.

Burghardt, G. M. and Greene, H. W. (1988). Predator simulation and duration of death feigning in neonate hognose snakes. Anim. Behav. 36, 1842-1844.

Cornwall, M. C., Ripps, H., Chappell, R. L. and Jones, G. J. (1989). Membrane current responses of skate photoreceptors. J. Gen. Physiol. 94, 633-647.

Cott, H. B. (1940). Adaptive Coloration in Animals. London: Methuen \& Co., Ltd.

Crook, A. C. (1997a). Colour patterns in a coral reef fish: Is background complexity important? J. Exp. Mar. Biol. Ecol. 217, 237-252.

Crook, A. C. (1997b). Determinants of the physiological colour patterns of juvenile parrotfish, Chlorurus sordidus. Anim. Behav. 53, 1251-1261.

Cuthill, I. C. and Székely, A. (2009). Coincident disruptive coloration. Philos. Trans. R. Soc. Lond. B 364, 489-496.

Cuthill, I. C., Stevens, M., Sheppard, J., Maddocks, T., Párraga, C. A. and Troscianko, T. S. (2005). Disruptive coloration and background pattern matching Nature 434, 72-74.

Denton, E. J. and Land, M. F. (1971). Mechanism of reflexion in silvery layers of fish and cephalopods. Proc. R. Soc. Lond. A 178, 43-61.

Douglas, R. H. (2018). The pupillary light responses of animals; a review of their distribution, dynamics, mechanisms and functions. Prog. Retin. Eye Res. 66, $17-48$.

Douglas, R. H., Harper, R. D. and Case, J. F. (1998). The pupil response of a teleost fish, Porichthys notatus: description amd comparison to other species. Vision Res. 38, 2697-2710.

Douglas, R. H., Collin, S. P. and Corrigan, J. (2002). The eyes of suckermouth armoured catfish (Loricariidae, subfamily Hypostomus): pupil response, lenticular longitudinal spherical aberration and retinal topography. J. Exp. Biol. 205 3425-3433

Douglas, R. H., Williamson, R. and Wagner, H.-J. (2005). The pupillary response of cephalopods. J. Exp. Biol. 208, 261-265.

Dowling, J. E. (2013). The Retina: An Approachable Part of the Brain. 2nd edn. Cambridge, MA: Belknap Press of Harvard University Press.

Dowling, J. E. and Ripps, H. (1970). Visual adaptation in the retina of the skate J. Gen. Physiol. 56, 491-520.

Ebert, D. A., Cowley, P. D. and Compagno, L. J. V. (1991). A preliminary investigation of the feeding ecology of skates (Batoidea: Rajidae) off the west coast of southern Africa. South Afr. J. Mar. Sci. 10, 71-81.

Einhäuser, W., Stout, J., Koch, C. and Carter, O. (2007). Pupil dilation reflects perceptual selection and predicts subsequent stability in perceptual rivalry. Proc. Nat. Acad. Sci. 105, 1704-1709.

Endler, J. A. (1978). A predator's view of animal color patterns. Evol. Biol. 11, 319-364.

Endler, J. A. (1981). An overview of the relationships between mimicry and crypsis Biol. J. Linn. Soc. 16, 25-31.

Endler, J. A. (1984). Progressive background matching in moths, and a quantitative measure of crypsis. Biol. J. Linn. Soc. 22, 187-231.

Gagliardi, G. J., Gallup, G. G. J. and Boren, J. L. (1976). Effect of different pupil to eye size ratios on tonic immobility in chickens. Bull. Psychon. Soc. 8, 614-616.

Gamlin, P. D. R., Zhang, H., Harlow, A. and Barbur, J. L. (1998). Pupil responses to stimulus color, structure and light flux increments in the rhesus monkey. Vision Res. 38, 3353-3358.

Hammond, P. and Mouat, G. S. V. (1985). The relationship between feline pupil size and luminance. Exp. Brain Res. 59, 485-490.

Hanlon, R. T. and Messenger, J. B. (2018). Cephalopod Behaviour. Cambridge: Cambridge University Press.
Hennig, C. W. (1977). Effect of simulated predation on tonic immobility in Anolis carolinensis: the role of eye contact. Bull. Psychon. Soc. 9, 239-242.

Hess, E. H. and Polt, J. M. (1964). Pupil size in relation to mental activity during simple problem-solving. Science 143, 1190-1192.

Jacobsen, I. P. and Bennett, M. B. (2013). A comparative analysis of feeding and trophic level ecology in stingrays (Rajiformes; Myliobatoidei) and electric rays (Rajiformes: Torpedinoidei). PLOS ONE 8: e71348.

Kelman, E. J., Baddeley, R. J., Shohet, A. J. and Osorio, D. (2007). Perception of visual texture and the expression of disruptive camouflage by the cuttlefish, Sepia officinalis. Proc. R. Soc. B 274, 1369-1375.

Land, M. F. and Nilsson, D.-E. (2012). Animal Eyes. Oxford: Oxford University Press Longley, W. H. (1916). Observations upon tropical fishes and inferences from their adaptive coloration. Proc. Natl. Acad. Sci. 2, 733-737.

Longley, W. H. (1917). Studies upon the biological significance of anima coloration. I. The colors and color changes of West Indian reef-fishes. J. Exp. Biol. 1, 533-601.

Mabragaña, E. and Giberto, D. A. (2007). Feeding ecology and abundance of two sympatric skates, the shortfin sand skate Psammobatis normani McEachran, and the smallthorn sand skate $P$. rudis Günther (Chondrichthyes, Rajidae), in the southwest Atlantic. ICES J. Mar. Sci. 64, 1017-1027.

Marshall, N. J. (2000). Communication and camouflage with the same 'bright' colours in reef fishes. Philos. Trans. R. Soc. Lond. B 355, 1243-1248.

Marshall, N. J., Jennings, K. J., McFarland, W. N., Loew, E. R. and Losey, G. S. (2003). Visual biology of Hawaiian coral reef fishes. II. Colors of Hawaiian coral reef fish. Copeia 3, 455-466.

Mäthger, L. M., Barbosa, A., Miner, S. and Hanlon, R. T. (2006). Color blindness and contrast perception in cuttlefish (Sepia officinalis) determined by a visua sensorimotor assay. Vision Res. 46, 1746-1753.

Mäthger, L. M., Denton, E. J., Marshall, N. J. and Hanlon, R. T. (2009) Mechanisms and behavioral functions of structural colouration in cephalopods J. R. Soc. Interface 6, S149-S163.

McEachran, J. D. and Musick, J. A. (1975). Distribution and relative abundance of seven species of skates (Pisces: Rajidae) which occur between Nova Scotia and Cape Hatteras. Fish. Bull. 73, 110-136.

Merilaita, S. (1998). Crypsis through disruptive coloration in an isopod. Proc. R. Soc B 265, 1059-1064.

Messenger, J. B. (1981). Comparative physiology of vision in molluscs. In Comparative Physiology and Evolution of Vision in Invertebrates. C: Invertebrate Visual Centers and Behavior II (ed. H. Autrum), pp. 93-200. Springer Verlag.

Muntz, W. R. A. (1977). Pupillary response of cephalopods. Symp. Zool. Soc. Lond. 38, 277-285.

Neudecker, S. (1989). Eye camouflage and false eyespots: chaetodontid responses to predators. Environ. Biol. Fishes 25, 143-157.

Nilsson, D.-E. and Nilsson, H. L. (1983). Eye camouflage in the isopod crustacean Astacilla longicornis (Sowerby). J. Exp. Mar. Biol. Ecol. 68, 105-110.

Orlov, A. M. (1998). On feeding of mass species of deep-sea skates (Bathyraja spp. Rajidae) from the Pacific waters of the Northern Kurils and Southeastern Kamchatka. J. Ichthyology 38, 635-644.

Parker, G. H. (1936). Color changes in elasmobranchs. Proc. Natl Acad. Sci. USA 22, 55-60.

Parker, G. H. and Porter, H. (1934). The control of the dermal melanophores in elasmobranch fishes. Biol. Bull. 66, 30-37.

Ramachandran, V. S., Tyler, C. W., Gregory, R. L., Rogers-Ramachandran, D. Duensing, S., Pillsbury, C. and Ramachandran, C. (1996). Rapid adaptive camouflage in tropical flounders. Nature 379, 815-818.

Ripps, H. and Dowling, J. E. (1990). Structural features and adaptive properties of photoreceptors in the skate retina. J. Exp. Zool., 46-54.

Robins, C. R. and Ray, G. C. (1986). A Field Guide to Atlantic Coast Fishes of North America. Boston: Houghton Mifflin Company.

Stavenga, D. G., Smits, R. P. and Hoenders, B. J. (1993). Simple exponentia functions describing the absorbance bands of visual pigment spectra. Vision Res. 33, 1011-1017.

Stevens, M. and Cuthill, I. C. (2006). Disruptive coloration, crypsis and edge detection in early visual processing. Proc. R. Soc. B 273, 2141-2147.

Stevens, M. and Merilaita, S. (2009). Animal camouflage: current issues and new perspectives. Phil. Trans. R. Soc. Lond. B 364, 423-427.

Stevens, M. and Merilaita, S. (2011). Animal Camouflage: Mechanisms and Function. Cambridge: Cambridge University Press.

Stevens, M., Cuthill, I. C., Windsor, A. M. M. and Walker, H. J. (2006). Disruptive contrast in animal camouflage. Proc. R. Soc. B 273, 2433-2438.

Stuart-Fox, D. and Moussalli, A. (2009). Camouflage, communication and thermoregulation: lessons from colour changing organisms. Phil. Trans. R. Soc Lond. B 364, 463-470.

Thayer, G. H. (1909). Concealing Coloration in the Animal Kingdom. An Exposition of the Laws of Disguise Through Color and Pattern: Being a Summary of Abbott H. Thayer's Discoveries. New York: The Macmillan Company.

Thresher, R. E. (1977). Eye ornamentation of Caribbean reef fish. Z. Tierpsychol. 43, $152-158$ 
Troscianko, T., Benton, C. P., Lovell, P. G., Tolhurst, D. J. and Pizlo, Z. (2009). Camouflage and visual perception. Phil. Trans. R. Soc. Lond. B 364, 449-461.

Visconti, M. A., Ramanzini, G. C., Camargo, C. R. and Castrucci, A. M. L. (1999). Elasmobranch color change: a short review and novel data on hormone regulation. J. Exp. Zool. 284, 485-491.

Vorobyev, M., Marshall, J. N., Osorio, D., Hempel de Ibarra, N. and Menzel, R. (2001). Colourful objects through animal eyes. Color Res. Appl. S26, S214-S217. Waring, H. (1963). Color Change Mechanisms of Cold-Blooded Vertebrates. New York, London: Academic Press.
Warrant, E. J. (2004). Vision in the dimmest habitats on Earth. J. Comp. Physiol. A 190, 765-789.

Warrant, E. J. and Nilsson, D.-E. (1998). Absorption of white light in photoreceptors. Vision Res. 38, 195-207.

Wilcox, J. G. and Barlow, H. B. (1975). The size and shape of the pupil in lightly anaesthetized cats as a function of luminance. Vision Res. 15, 1363-1365.

Zylinski, S. and Johnsen, S. (2011). Mesopelagic cephalopods switch between transparency and pigmentation to optimize camouflage in the deep. Curr. Biol. 21, 1937-1941. 